\title{
L'HYDROPTERE: HOW MULTIDISCIPLINARY SCIENTIFIC RESEARCH MAY HELP BREAK THE SAILING SPEED RECORD
}

\author{
M Calmon, M Farhat, P Fua, K Startchev, G Bonnier, J-A Månson, V Michaud, A Sigg, M Oggier, MO Deville, \\ O Braun, ML Sawley, L Blecha, J Cugnoni, Ecole Polytechnique Fédérale de Lausanne (EPFL), CH \\ JM Bourgeon, S Dyen, D Moyon, D Schmäh, R Amacher, D Colegrave, Hydroptère Design Team
}

\section{SUMMARY}

In 2009, l'Hydroptère broke the symbolic barrier of 50 knots and became the world fastest sailing boat over both 500 meters and 1 nautical mile. This major achievement relied on the high skills of the sailing team but also on technical advances of the boat, resulting from the scientific collaboration between the Hydroptère Design Team and the Ecole Polytechnique Fédérale de Lausanne (EPFL). In the present article, we highlight the multidisciplinary research activity performed within EPFL in the course of this collaboration involving aero- and hydrodynamics, materials and structure as well as computer vision. Various foils were tested at reduced scale in a high speed water tunnel, and the results used to validate the numerical simulations. Composite materials, their processing parameters and assembly components were tested. The structural behaviour was also investigated to determine strains and stresses in normal and extreme sailing conditions, taking waves into account, and a combined model was derived for dynamic simulation. Finally, advanced computer vision methods were developed and implemented on the boat to monitor foil immersion and cross beams deformations.

\section{NOMENCLATURE}

$C_{\mathrm{D}}$

$C_{\mathrm{L}}$

$d$

$G_{\text {Ic }}$

$\theta$

$P, Q$

$p$

$p_{\mathrm{v}}$

$\rho$

$\sigma$

V

2D

3D

CFD

CVLab

DMA

DSC

EASY

EPFL

FE

HPC

LES

LIN

LMAF

$\mathrm{LMH}$

LTC
Angle of attack, angle of yaw $\left(^{\circ}\right)$

Drag coefficient

Lift coefficient

Level of immersion (m)

Griffith's critical strain energy release rate $(\mathrm{J})$

Angle of refraction $\left(^{\circ}\right)$

Pixel distributions

Static pressure $(\mathrm{Pa})$

Vapour pressure of water (2300 Pa)

Density of water $\left(997 \mathrm{~kg} \mathrm{~m}^{-3}\right)$

Cavitation number $\left(=\frac{p-p_{v}}{\frac{1}{2} \rho V^{2}}\right)$

Flow velocity $\left(\mathrm{m} \mathrm{s}^{-1}\right)$

Two-dimensional

Three-dimensional

Computational Fluid Dynamics

Computer Vision Laboratory

Dynamic Mechanical Analysis

Differential Scanning Calorimetry

Evolutionary Algorithm SYstem

Ecole Polytechnique Fédérale de

Lausanne

Finite Element

High-Performance Computer

Large Eddy Simulation

Computational Engineering Laboratory

Laboratory of Applied Mechanics and

Reliability Analysis

Hydraulic Machines Laboratory

Laboratory of Polymer and Composite

Technology

$\begin{array}{ll}\text { NTUA } & \begin{array}{l}\text { National Technical University of } \\ \text { Athens }\end{array} \\ \text { PIV } & \text { Particle Image Velocimetry } \\ \text { RANS } & \text { Reynolds Averaged Navier-Stokes } \\ \text { SST } & \text { Shear Stress Transport } \\ \text { UD } & \text { Unidirectional tape } \\ \text { UFO } & \text { Unidentified Floating Object } \\ \text { URANS } & \text { Unsteady RANS } \\ \text { VOF } & \text { Volume Of Fluid }\end{array}$

\section{INTRODUCTION}

L'Hydroptère is a sailing trimaran using the lift of hydrofoils in a whole sense, i.e. using them to balance completely the weight of the boat and to pull the hulls out of the water from a minimum boat speed, thus reducing drastically hydrodynamic drag and raising performance to high levels.

L'Hydroptère already has a long history of developments, records and breakages. Mentioning only the most recent and symbolic, in 2005 she crossed the Channel faster than aviator Louis Blériot in 1909, sailing from Dover to Calais in 34 minutes and 24 seconds, at an average speed of over 33 knots. She then unfortunately collided with an Unidentified Floating Object (UFO) during a Cadiz to San Salvador crossing record attempt. Afterward, Swiss nationals Thierry and Adrien Lombard saved the project and established a contact between the Hydroptère Team and the Ecole Polytechnique Fédérale de Lausanne (EPFL), giving rise to a scientific partnership in 2007. This was accompanied by a move towards outright sailing speed records in inshore conditions. Finally, with step-by-step improvements on the boat, increasing velocity levels were undertaken from 2007 to 2009 , until the recent successes of the two new 
world records over $500 \mathrm{~m}$ and 1 nautical mile respectively of 51.36 knots and 50.17 knots.

To achieve such outstanding performance with wind as the only driving force, the Hydroptère Team has required to decrease air resistance of all the boat's aerodynamic elements and to improve both shape and tuning of the sails in order to obtain as much propulsive force as possible. But the main challenge is to obtain sufficient lift force from the hydrofoils despite phenomena encountered at high speed like cavitation and ventilation that can lead to hydrodynamic load instabilities. Moreover, structural design, materials choice and manufacture are the main factors affecting the safety and stability of the yacht and can significantly affect its overall performance. In such a trans-disciplinary project, the global design and optimization process usually requires a significant number of inputs and hypotheses coming from different fields like fluid dynamics for the determination of the wide range of loading conditions, or materials science and solid mechanics for the determination of the failure criteria of the materials that can be used. Knowing these design criteria, an iterative design-simulation-optimization process can then be employed to progressively turn an innovative design concept into a highly sophisticated and high-performance hydrofoil yacht (Figure 1).

Several laboratories at EPFL have contributed to the optimization of l'Hydroptère to achieve the world sailing speed records; but also contributed to the design of the smaller l'Hydroptère.ch catamaran that will soon be launched on Lake Geneva. Several Finite Element (FE) models of the structure have been developed, and updated for l'Hydroptère. Specific materials have also been developed, produced and tested in various conditions within the aim to optimize the structural behaviour and provide new design solutions. Onboard measurements of foil immersion have also been used to validate and refine the design loads and operation scenarios and proved to be very important in the fine optimization of the yacht. Various foils have been tested at reduced scale in a high speed water tunnel, and the results used to validate the numerical simulations.

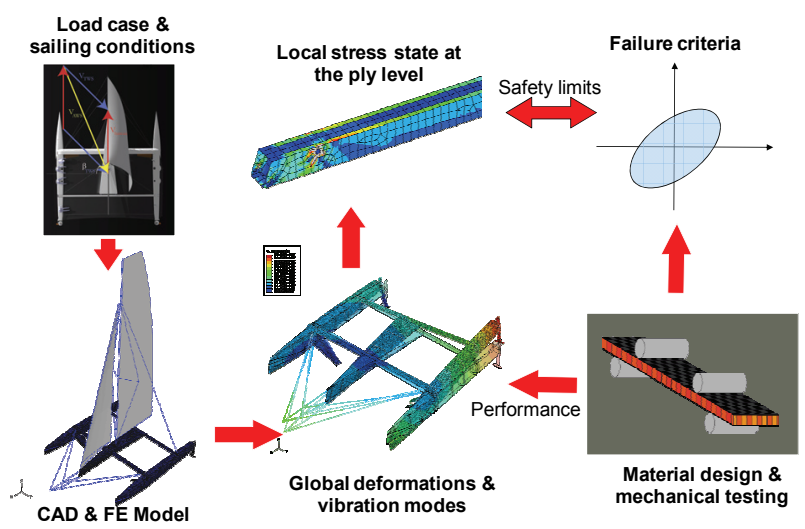

Figure 1: The global design \& optimization process used for l'Hydroptère

\section{PROBLEM STATEMENT}

Contrary to an Archimedean yacht, while flying over the water surface, l'Hydroptère must constantly be maintained in equilibrium between two dynamic fluidstructure interaction forces; namely the hydrodynamic forces on the foils and the aerodynamic forces of the sails. Between the aero- and hydrodynamic loading points, the structure must transmit all the efforts and be as lightweight as possible while preserving a reasonable safety factor. However, due to the localized hydrodynamic loading and the narrow working range of angle of attack of the hydrofoils, the stiffness of the platform is much more important than for more traditional multi-hull yachts. Indeed, if the cross beams supporting the hydrofoils are deformed by only a few degrees, the flow condition around the foil can change dramatically. Ideally the torsion of the cross beams should be significantly lower than the working range of angle of attack of the hydrofoil to avoid any coupling between structural deformations and the hydrodynamic behaviour of the foils. Additionally, considering now the dynamics of the yacht, the first eigen frequencies of the structural deformation would be required to be much higher than the frequencies of the hydrodynamic flow around the foil in order to obtain the best performance of the hydrofoils in all conditions. Constructing such a stiff structure is however contrary to the principle of minimization of the weight and thus these conditions are usually impossible to achieve and are anyway not optimal. In most cases, the hydrodynamic flow conditions of the foils and the structural deformations cannot be decoupled in a static and / or a dynamic sense. Thus, both the structural deformations and the hydrodynamic response of the foils must be taken simultaneously into account in the models used for the design and optimization of a high performance hydrofoil yacht.

\section{DYNAMIC SIMULATION}

Existing fluid-structure interaction simulation methods can provide detailed predictions of both physics and to a certain extent predictions of fluid-structure instabilities. However, most of these methods are based on a full or iterative coupling of a three-dimensional (3D) Reynolds Averaged Navier-Stokes (RANS) solver with a 3D structural FE model. Due to the high cost of the 3D RANS fluid flow solution and the high number of iterations required to solve an unsteady fluid-structure problem, these methods cannot be reasonably applied to the design \& optimization of a large scale structure like l'Hydroptère. To study this coupled fluid-structure problem in a more computationally efficient way, a specific simulation method has been developed in the Laboratory of Applied Mechanics and Reliability Analysis (LMAF). 
The basic assumption of the developed computational method is to consider that the global dynamics of the structure (a few $\mathrm{Hz}$ in general) is much slower than the dynamics of the flow around the hydrofoils and thus that the fluid flow can be considered stationary at all time. A reduced parametric model of the hydrodynamic pressure distribution on the foil has been established using a series of steady Computational Fluid Dynamics (CFD) simulations carried out with ANSYS CFX [1]. A continuous hydrodynamic response surface has then been derived using various polynomial interpolation functions between the discrete set of calculated flow conditions. This parametric hydrodynamic force model has then been implemented in a user defined finite element formulation in the structural FE code Dassault Systèmes SIMULIA Abaqus [2] in order to represent the evolution of the pressure distribution as a function of the current navigation conditions (velocity, local effective angle of attack and foil immersion) and structural deformations (Figure 2).

Thanks to the versatility of the method, the developed hydrodynamic surface finite element has been used to calculate static deformation and stresses in given sailing condition, extract the resonant frequencies and modes of vibration of the structure and simulate the transient behavior of the platform while crossing a front of waves.

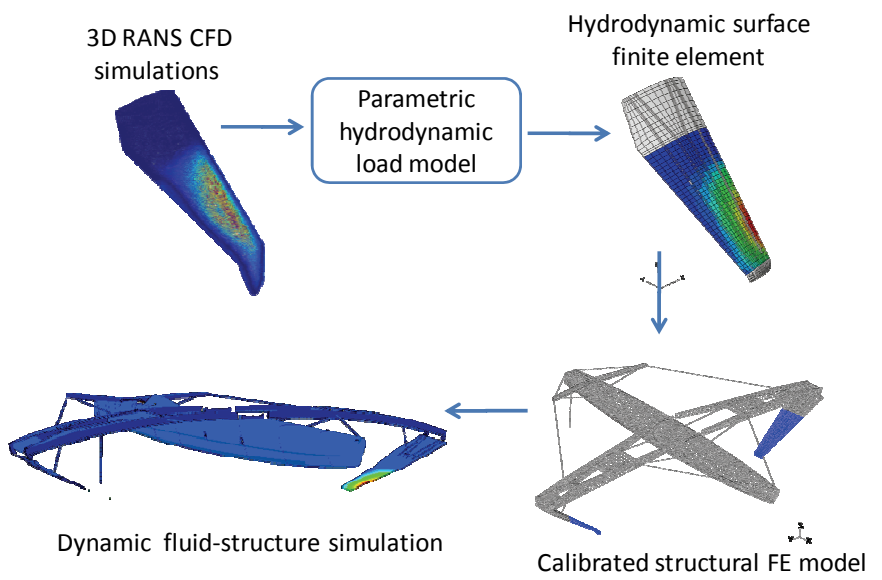

Figure 2: A schematic of the developed fluid-structure simulation method for l'Hydroptère

\section{STRUCTURE}

For precise predictions of the dynamic behavior of the yacht, a detailed and accurate model of the structure is required. However, because of the large number of variables (material properties, geometry and assembly) and their inherent uncertainties, the developed FE model had to be thoroughly validated on the basis of real structural tests. A series of tests have been conducted first on components and then on the whole yacht. The static deformations of key elements were measured under various loading conditions to determine the most important stiffness values and a detailed modal analysis of the foils alone and the whole yacht was carried out. A specific optical displacement measurement procedure based on digital image correlation was developed to measure small deformations and rotations on such a large structure, with resolution of $\sim 0.1 \mathrm{~mm}$ or $0.01^{\circ}$ at $10 \mathrm{~m}$ distance (Figure 3).
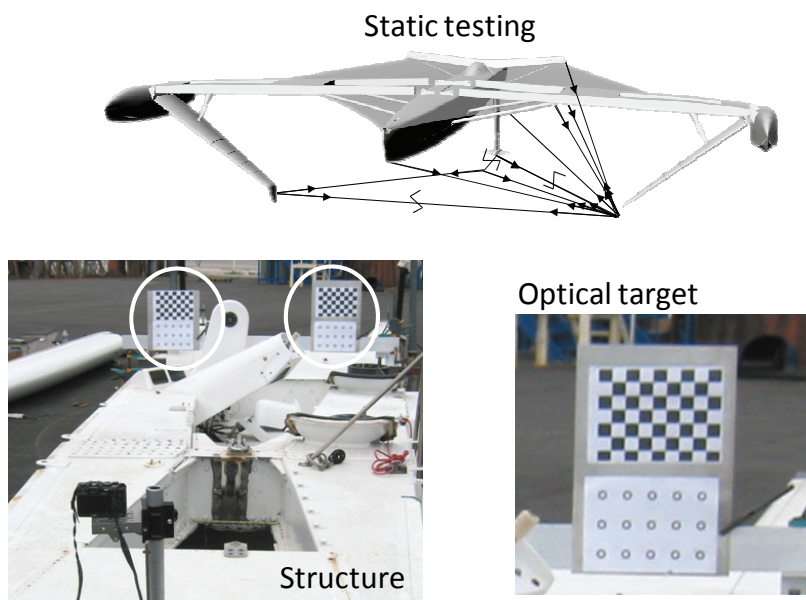

High res. camera

Figure 3: Static tests of l'Hydroptère platform using a custom developed digital image correlation displacement measurement technique

The measured stiffness's and Eigen frequencies were then used to update the FE model parameters in order to achieve a good agreement between the numerical predictions and the real behaviour of the structure. A FE model optimization method was developed and interfaced with the Abaqus solver to automate the parameter updating procedure and maximize the accuracy of the numerical model (Figure 4).

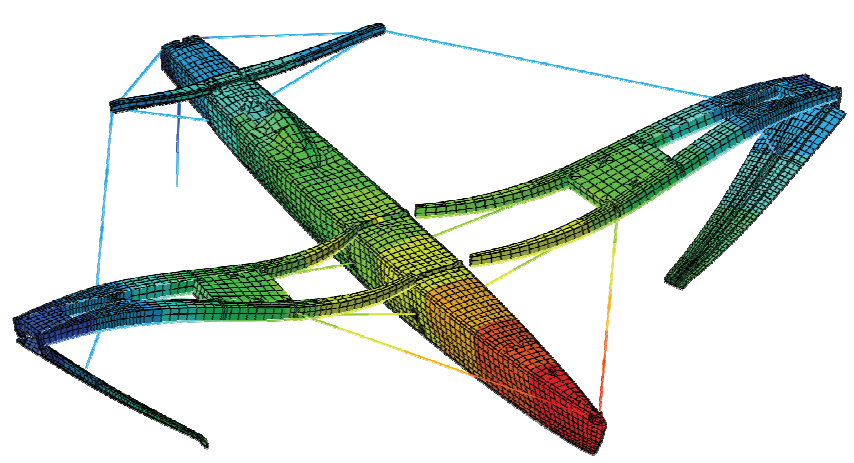

Figure 4: Numerical modal analysis of the whole l'Hydroptère structure using the calibrated model

The calibrated FE model was also used to provide important modal analysis data to the Hydroptère Design Team in order to study the hydrodynamic stability (divergence / flutter) of the rear stabilizator at high speed (Figure 5). 

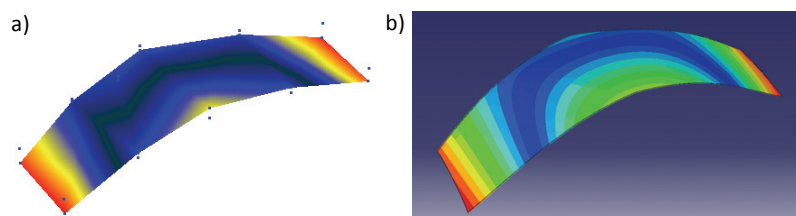

Figure 5: Experimental modal analysis of the rear horizontal stabilizator (a) and predictions using the calibrated model (b)

\section{ONBOARD MEASUREMENT SYSTEM}

\subsection{PRESENTATION}

A measurement system on board provides the sailors with real time values and is divided into four subsystems:

- Stress and positioning sensors

- Navigation unit

- Inertial unit

- Video system

The stress measuring and positioning system is composed of 54 sensors, placed on various strategic points on the boat. It includes four types of recordings:

- Strain gauges, measuring local stress, which permit the analysis of the efforts sustained by the structure of the boat

- Accelerometers

- Rotation sensors

- Pressure sensors, particularly in the jacks mounted as struts between the foils and the cross-beam.

All these sensors are connected to HBM digiCLIP digital amplifiers.

The navigation unit indicates the environment in which l'Hydroptère evolves, speed, angle, direction of real and apparent winds, and the GPS positioning of the boat. As well as for the whole marine electronic system, it is provided by $\mathrm{B} \& \mathrm{G}$, with its upper class WTP2 processor.

The IXSEA Octans inertial unit gives the boat's attitudes, roll, pitch, surge, yaw and dig in, as well as their speeds and accelerations, with great precision.

The video system has been added using Cosworth Pi VIDS2 video recorder, and a few cameras, in order to analyse in real time the submerged depth of immersed appendages, as well as the structure deformation, with video-imaging techniques.

All the values from the four sub-systems are spread in a CAN bus and recorded in a synchronised way into a logger box (Cosworth Pi Sigma LLB data logger), as well as copied to a ruggedized computer (Lemer Pax Posibox) in order to:

- Display with visual and sound effects to inform the test engineer on the applied strain, with an alarm signal if necessary

- Process the data through basic mathematical tools or more powerful tools such as filtering or the Fourier transform.

The real-time data are an essential aid to steer the carbon bird over the irregular air-water interface and after every sail test the collected data is analyzed to make improvement for the next sailing day. Those recordings could also be compared off-line to the estimates of the flight simulator, and serves as a database for improvements or new designs.

\subsection{COMPUTER VISION EXTENSION}

\section{2 (a) Foil Immersion Detection}

Only the resultant of the hydrodynamic loads on the foil is deduced from the jack hydraulic pressure. This resultant is the product of the hydrodynamic pressure distribution over the submerged surface of the foil. Thus, high load over only a tip of the foil could lead to the same jack load than a smaller load spread over the whole foil. The knowledge of the submerged depth of the foil allows refining the true effect of hydrodynamic loads.

The principle of the foil immersion detection was derived at the Computer Vision Laboratory (CVLab). The definition of the level of immersion uses the refraction phenomena. It consists in moving along the edge of the foil, and looking for a change of slope due to the refraction of the submerged part (Figure 6). The method, simple in its principle, could be quite challenging in its application since a lot of perturbations could disturb the detection: changing light condition, reflections or spray drops on lens resulting in blurred images.

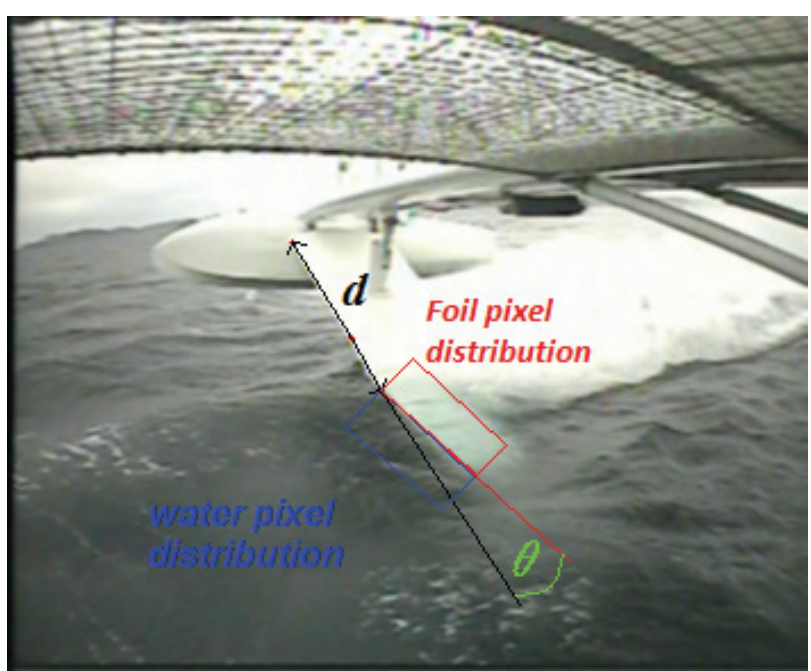

Figure 6: Definition of level of immersion using refraction phenomenon and Kullback-Liebler divergence 
The algorithm is based on the maximization of a function of the Kullback-Liebler divergence [3] between pixel distributions $P$ and $Q$ of the non-submerged or submerged parts of the foil, and water (histogram of pixels), given a level of immersion $d$ and an angle of refraction $\theta$ :

$$
\begin{aligned}
F(d, \theta)= & \frac{D_{K L}(P, Q)+D_{K L}(Q, P)}{2} \\
& =\frac{1}{2} \sum_{0}^{255} P(i) \log \frac{P(i)}{Q(i)}+Q(i) \log \frac{Q(i)}{P(i)}
\end{aligned}
$$

The results from this tool can be quite interestingly compared with other synchronised measures (Figure 7).

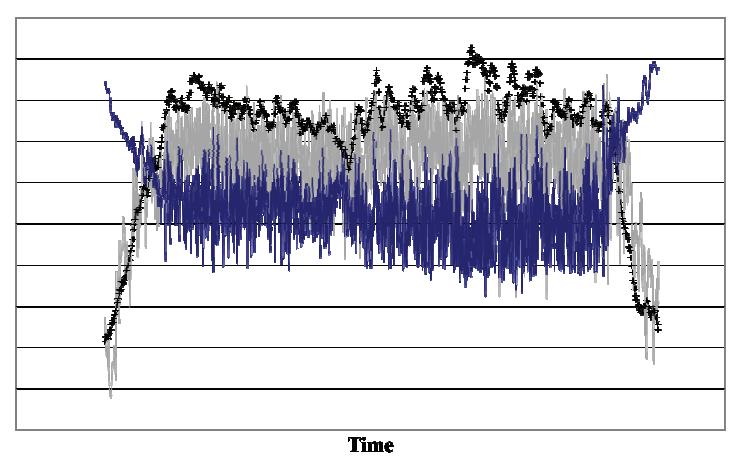

Figure 7: Time synchronized correlation between boat speed $(+)$, jack load (dark blue) and level of immersion (gray)

\section{2 (b) Cross-Beam Torsion Detection}

The ship platform is so flexible that it is not enough to monitor strain gauges in strategic points, but it is necessary to identify the greatest deformations of the structure. The main deformation could be the cross-beam torsion since it modifies the foil angle of attack, and thus the hydrodynamic loads. This deformation could also play a crucial role in flight instabilities events.

Again, a computer vision tool is developed at CVLab. The principle is based on the minimization of a function using the difference between probability images, generated from the recorded images, and a linear combination of constructed images from a database. This database is a basis build up from model images of the same view as from the camera, using the deformations modes of the finite element structural model of the ship platform. Choosing a basis that underlines the in-view deformations in the modes allows identifying for example the cross-beam torsion, as a parameter in the function to minimize.

\section{MATERIALS}

To minimize the weight of the structure while answering to the needs of the design, it is necessary to carefully evaluate and optimize the properties of materials and of their assembly in conditions that are very close to ship yard practice (humidity, temperature, lay-up and processing time, part shape, etc...). Several studies were thus performed to gain confidence and potentially reduce safety factors in the part design.

\subsection{MANUFACTURING PROCESSES}

Most parts of the boat are made of carbon-epoxy composites, either as monolithic parts or as sandwich structures with honeycomb cores. Several processing methods are used, depending on the part design, from lay-up of wet-impregnated fabrics or prepregs cured under vacuum bag only, to prepregs cured in an autoclave. For all these, several studies were carried out to select the best process, including the cure schedule, and to check the part quality after processing. Additional studies were carried out to analyse the influence of the off-axis plies in the final part properties in bending. Occasionally, analyses were carried out on processed parts, especially if potential defects are suspected: visual inspections by micrographs or curing stage by Differential Scanning Calorimetry (DSC) and Dynamic Mechanical Analysis (DMA) for example. A constant interaction between the boat builders, the Hydroptère Design Team and the lab is crucial to obtain best results.

\subsection{SANDWICH STRUCTURES}

A specific study was carried out to compare the design values with experimental results for honeycomb sandwich structures processed with Nomex Flexcore under vacuum bag only (Figure 8). The final aim was to determine how this anisotropic honeycomb affects the properties of the final sandwich of the hull and provided experimental values for simulation model.

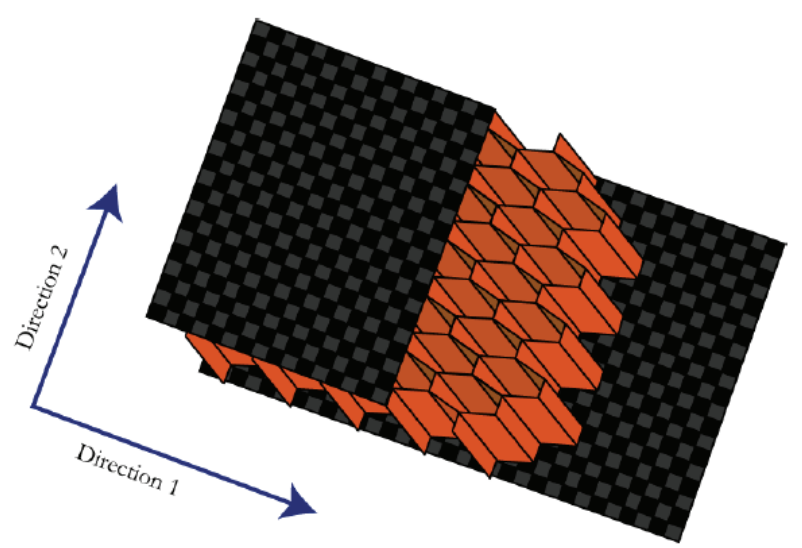

Figure 8: Anisotropy of the Nomex Flexcore and the two main directions

For this, samples consisting of skins only, then full sandwich structures were manufactured and tested in tension, compression and shear for the skins, and in four points bending with several span lengths for the sandwich beams in order to calculate the shear modulus of the core in the two main directions. A careful 
comparison of the experimental values, with the estimated values from the skins and core properties was carried out and used in the simulated model.

\subsection{THREADED JOINTS}

Several metallic parts are attached to the composite parts through threaded joints, for example the mast railing. A study was conducted to assess the strength of these joints, as a function of the composite type (unidirectional UD vs. quasi-isotropic), the screw material (stainless steel vs. composite), the presence of an insert or not, and how it is assembled (glued screw, screw with release agent and glued). Joints were made, and a testing device was produced that allowed the screws to be tested by extracting them with a force perpendicular to the composite plane. Results show as expected that it is important to reinforce the joint location by quasiisotropic lay-up (Figure 9). Also, direct threading with a proper tool, with a glue layer, gives the best results. However, a request could be that joints can be removed easily for dismounting and repair. The use of a release agent before gluing the screw does not decrease the ultimate strength, even if the screw is un-screwed and rescrewed. These results do not take into account aging effects. If the screw is to be removed "frequently", a glued insert can be safely used, albeit with a weight penalty.

(a)

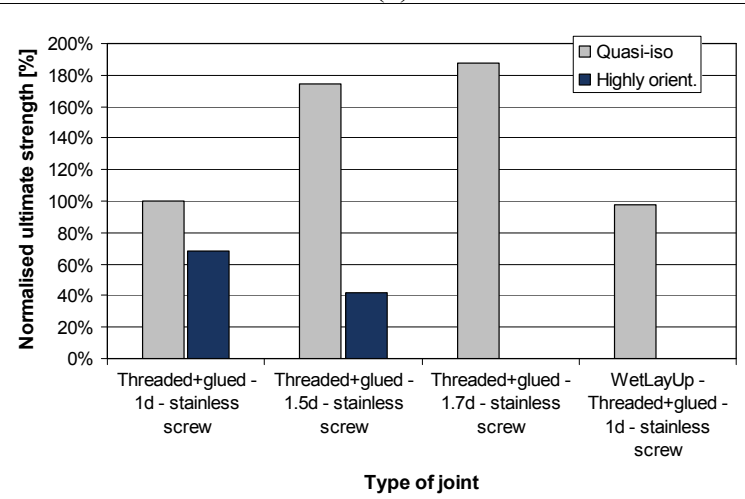

(b)

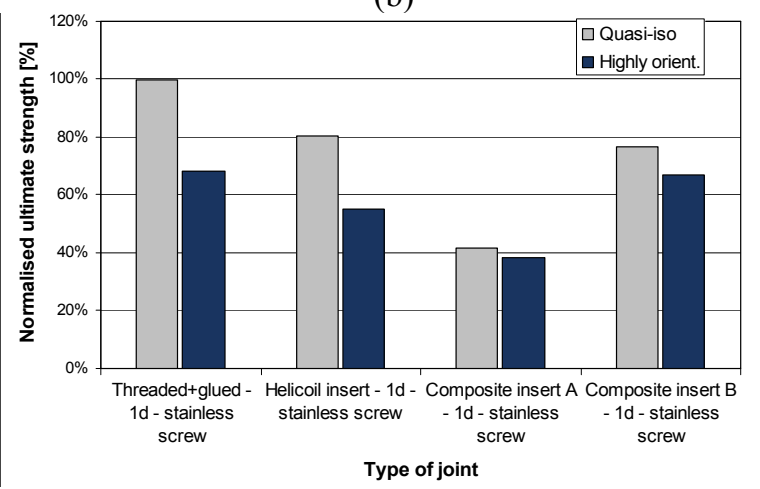

Figure 9: Comparison of the pull-out force for several types of joint (a) Thread length effects, (b) Thread type effects $(100 \%$ reference value correspond to stainless steel screw threaded in quasi-isotropic laminate, with embedment length of the screw diameter)

\subsection{BONDING}

An extensive study was carried out to optimise the surface treatment of titanium for bonding to composite parts. This is crucial to make sure these bonds are designed properly, and the effect of aging is taken into account. For this, comparative measurements of fracture strength and Griffith's critical strain energy release rate $G_{\text {Ic }}$ were carried out on adhesive bonded joints with three surface treatments of titanium. Tests were performed unaged and along thirteen weeks of accelerated aging in salted or de-ionized water at $50^{\circ} \mathrm{C}$. Thermo-mechanical measurements were carried out on the epoxy Araldite 420 adhesive alone, with the same aging conditions.

From these results (Figure 10), the best practical solution for the performance and lifespan of the bonded joint was determined. A combined surface treatment of sanding, degreasing and chemical etching shoed the best durability, whereas a treatment using a sulphuric anodic oxidation in addition showed the best adherence before aging. On the whole, bonded joints showed a high variability and a fall of their properties from 30 to $70 \%$ at the end of aging according to the surface treatment.

A numerical method for dimensioning was proposed by means of a finite element implementation with a cohesive zone model in Abaqus. This method was validated by simulation of two representative mechanical tests corroborating the experimental results. Experimentally determined failure criteria as well as aging effects and safety factors were thus taken into account in the finite element model.

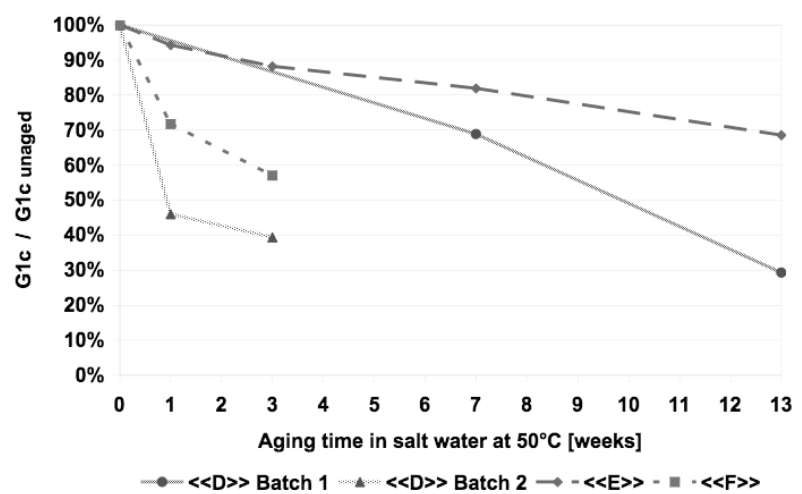

Figure 10: Mode I fracture energy after aging relative to the un-aged value in the three points bending tests for the three best surface treatments. D is sandblasted, degreased and etched; $\mathrm{E}$ is sandblasted, hot degreased and etched; F is bead blasted, degreased, etched and anodized.

\section{HYDRODYNAMICS}

\subsection{HYDRODYNAMIC PHENOMENA}

\section{1 (a) Cavitation}

The cavitation phenomenon is the formation of vapour cavities within a flowing liquid due to excessive decrease 
of local pressure [4]. It may occur in a variety of hydraulic systems such as hydraulic turbines and pumps, ship propellers and space rocket inducers. Cavitation may be the source of severe erosion and vibration as well as alteration of hydrodynamic performances.

In the specific case of 1'Hydroptère riding at 50 knots, it is almost impossible to avoid cavitation occurrence. Nevertheless, we have worked out the design of the foils to mitigate the cavitation consequences. In fact, as the boat speed is increased, cavitation may suddenly appear with a drastic drop of the hydrodynamic lift and a substantial increase of drag and structural vibrations. In this case, the safety may be seriously compromised.

In order to investigate the cavitation phenomenon in the case of l'Hydroptère foils, laboratory tests were carried out at the Hydraulic Machines Laboratory (LMH). 1/10 scaled models of both foils and rudder/stabilizer portions were mounted in the middle of $150 \mathrm{~mm} \times 150 \mathrm{~mm}$ square test section of the EPFL high speed cavitation tunnel (Figure 11). A maximum speed of $50 \mathrm{~m} / \mathrm{s}$ may be reached at the test section inlet and the pressure may be adjusted from 0.02 to $1.6 \mathrm{MPa}$. Cavitation can then be easily controlled, either enforced or avoided.

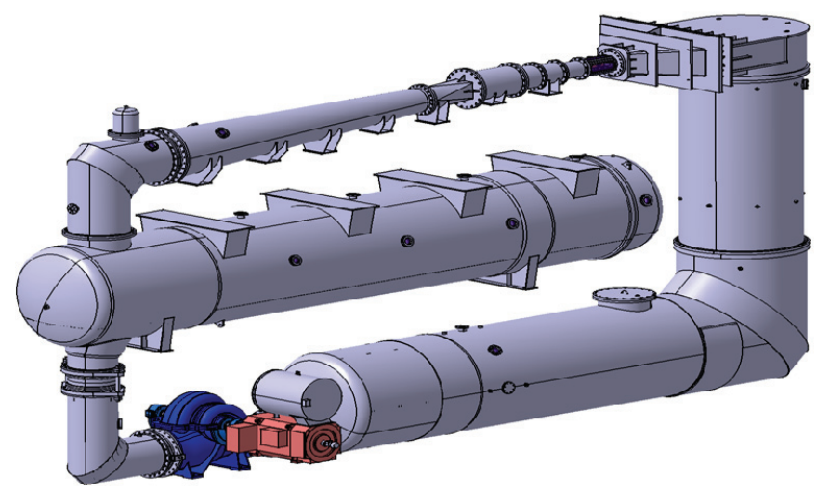

Figure 11: EPFL high speed cavitation tunnel [5]

To allow for accurate scale up, the flow velocity was set to $15 \mathrm{~m} / \mathrm{s}$ and the $\sigma$ value corresponded to l'Hydroptère speed of 50 knots under atmospheric pressure. For these conditions, a turbulent boundary layer develops on the foil and Reynolds effects may be neglected.

As illustrated on Figure 12, attached cavitation occurs on the foil. The location of the cavity detachment and its length depend strongly on the angle of attack. For low angles (left), cavitation departs downstream to the leading edge and extends rapidly beyond the trailing edge. In this regime, the flow is rather "smooth" with low induced vibration. As the angle is increased (right), the cavitation appears even for higher $\sigma$, and its detachment moves upstream with growing amplitude of the cavity pulsation. The sheet cavitation turns into the so called cloud cavitation, which is associated with large lift fluctuation and induced vibration.
In the design process, profile sections, distribution and angles of attacks of the foils of l'Hydroptère were optimized to avoid the occurrence of cloud cavitation.

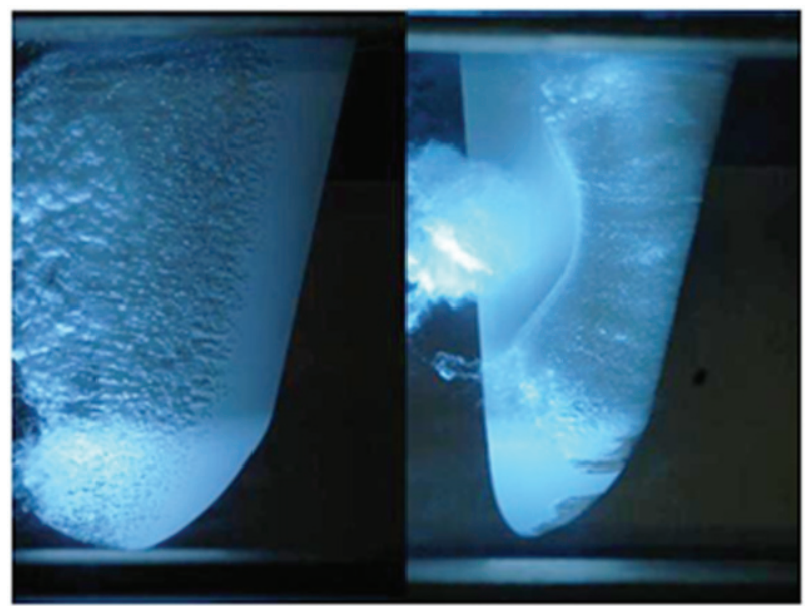

Figure 12: Cavitation trends

(Left: low $\alpha$ and $\sigma=0.26$;

Right: high $\alpha$ and $\sigma=0.87$ )

\section{1 (b) Ventilation}

Ventilation is a phenomenon in which air from above the free surface is sucked into a low pressure zone below the surface. It is of course more important in very low pressure zones, such as those that can lead to cavitation, and both phenomena are thus strongly interacting. Most often, air from above the surface is guided along the suction side, leading to a drop in lift (as well as in drag, although this is of lesser importance for hydrofoils) [6].

Unfortunately, the EPFL hydrodynamic tunnel is not suitable for ventilated tests. In fact, really few towing tank can reach the high speed required to reveal interesting effects.

\subsection{NUMERICAL SIMULATIONS}

Besides relatively standard numerical simulations using a 3D RANS solver and single phase models (as previously mentioned in the context of global design and optimization of l'Hydroptère, more advanced simulations were conducted with multi-phase models in order to deal with more local design and optimization problems, i.e. for the foils or rudder/stabilizer with respect to cavitation and ventilation phenomena. Two commercial finite volume solvers were used for this study: ANSYS FLUENT and ANSYS CFX [1]. Different approaches exist for the simulation of multi-phase flows, but Eulerian methods have been preferred. Both solvers have implemented a Volume of Fluid (VOF) method, with different specificities mainly for the interface or free surface computation. In both cases, a Realizable $k-\varepsilon$ turbulence model was used.

Using FLUENT, cavitation was initially investigated by simply "contouring" the low pressure zones below the 
vapour pressure; this approach provided interesting but only qualitative results. Another possible approach, using a "mixture" model did not provide adequate results, with a clear tendency to predict very large cavitation zones. More successful was the use of CFX with a three phase flow model. For ventilation, both solvers appeared to perform well, although the convergence properties of CFX were found to be better.

\subsection{VALIDATION OF SIMULATIONS}

Model scale tests involving three different phases of fluids (air, liquid water and water vapour) are extremely difficult to perform. For validation purposes, phenomena were divided and simulation directed towards two-phase flow cases: cavitation or ventilation alone.

\section{3 (a) Cavitation}

Numerical simulations of cavitation phenomenon were validated using experimental work carried out at the LMH in its high speed hydrodynamic tunnel. Initial validation studies were based on direct visual comparison between experimental photos and simulated images for the same flow conditions. Although this is only qualitative, it already gives a good idea of the ability to capture the presence of cavities, their extent and shape, the inception trends, etc.

More quantitative validation was performed through the variation of hydrodynamic loads according to cavitation extent. The experimental loads were determined by mounting the foil on a calibrated 5-axis balance, and compared with the simulated loads. For a cavitation inception of the type of Figure 12 left, Figure 13 indicates that the dependences of lift with moderate angle of attack are in agreement both with and without cavitation.

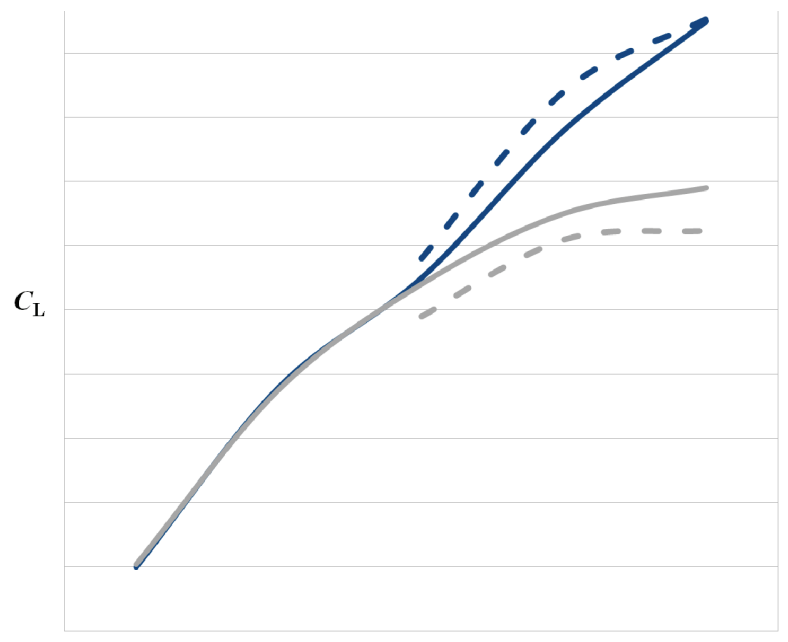

$\alpha$

Figure 13: Lift coefficient evolution vs. angle of attack; with high $\sigma=0.49$ and low cavitation (dark blue) or with low $\sigma=0.31$ and strong cavitation (gray);

(- - Simulation, - Experiments)

\section{3 (b) Ventilation}

Ventilation is a more complex phenomenon to validate, since the hydrodynamic tunnel is not suitable for ventilated tests. In fact, really few towing tank can reach the high speed required to reveal interesting effects.

The first step was again directed to visual comparisons to obtain a qualitative assessment, but on the true scale of the prototype. The shape of the wave elevation around the real foils was compared with pictures taken from onboard video recording system.

A second step was the quantitative comparison of load variation. Since no new and dedicated experimental tests could be done, an existing case study [6] was used. This book provides a short summary of experiments made in 1953 by Dingee at Davidson Laboratory (Stevens Institute of Technology, Hoboken, NJ, USA). The experiments employed a slender surface-piercing strut (lenticular section type with $12 \%$ relative thickness), with finite length and rounded lowered end. This body shape is closely representative of rudders, daggerboards or hydrofoils. The strut has a $63.5 \mathrm{~mm}$ chord with an initial immersion level of $63.5 \mathrm{~mm}$ (water free surface at rest). The strut was tested in a towing tank at a velocity of $7.62 \mathrm{~m} / \mathrm{s}$. Ventilation suddenly takes place at an angle of yaw around $15^{\circ}$, and lift reduces considerably while drag decreases slightly.

FLUENT exhibited convergence problems for flow around a finite length foil associated with complex 3D effects at the foil tip. However, successful computations were possible using CFX, with the results obtained being in excellent agreement with the experimental data (Figure 14).

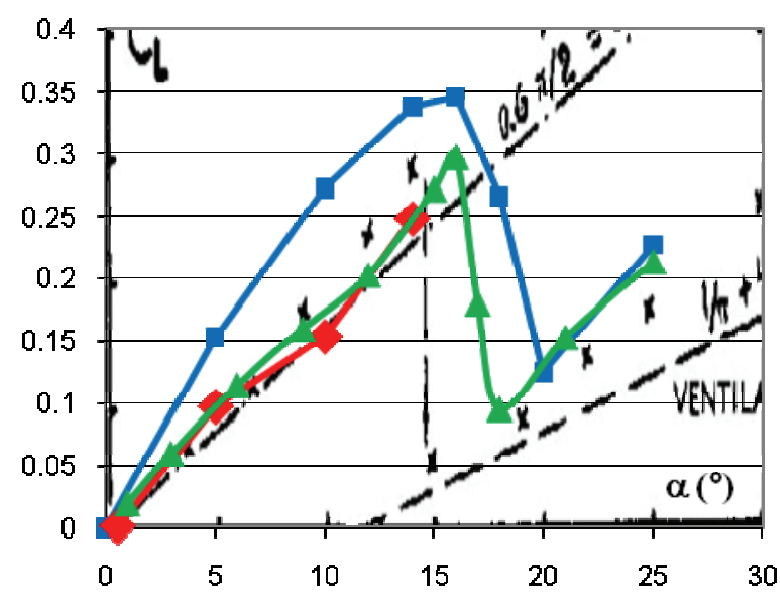

Figure 14: Lift coefficient of a surface-piercing strut as a function of angle of yaw

(a- FLUENT, strut with infinite length,

$\checkmark$ - FLUENT, strut with finite length,

$\Delta-$ CFX, strut with finite length, $\mathbf{x}$ - Dingee experiment [6]) 
7.4 OPTIMIZATION

\section{4 (a) Optimization of Steady Flow}

Fluid flow simulations offer the possibility to evaluate the performances of new designs quickly, enabling engineers to predict the influence of potential modifications on the boat performances.

As a first step, the validated models were used together to simulate more realistically the sailing conditions. Three-phase models using CFX were considered, but with a flat free water surface and steady conditions (Figure 15). An iterative manual optimization process could be performed by looking for foils with high lift-todrag ratio and low tendency to cavitation and ventilation.

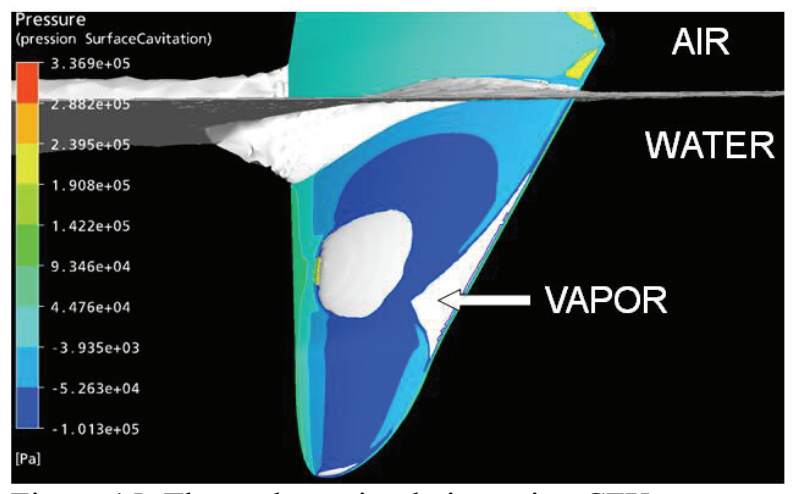

Figure 15: Three-phase simulation using CFX

\section{4 (b) Optimization Of Unsteady Flow}

The above-described optimization process, being overly simplified, could lead to extreme design solution. In fact, unsteady wind conditions and associated water surface waves and fluctuating trim angles of the boat result in a variable foil angle of attack. Peaks in the angle of attack can give rise to flow separation and stall of the hydrofoil, possibly further aggravated by ventilation on the submerged suction side. A low tendency to flow separation at high incidence angles should therefore be incorporated into the design criteria.

The Computational Engineering Laboratory (LIN) has undertaken a true multi-objective optimization procedure. Since such a procedure in principle involves both a large number of profiles and the requirement of unsteady conditions, further simplifications of the triphase flow over the foil is required. This was achieved by considering a two-dimensional (2D) hydrofoil in a rectangular test section with a variable angle of attack (pitch motion) and constant upstream velocity (single phase flow). Three optimization criteria were required to be maximized. The first is lift-to-drag ratio in nominal conditions $\left(C_{\mathrm{L}}=0.7\right)$. The two next criteria are the angle of attack margins from the angle of nominal condition until exceeding threshold values for the following two volumes:
- the first volume is related to flow separation, and thus is the extent of the zone of negative $x$ component of the flow velocity (with positive $x$ as flow direction),

- the second volume, in order to account for cavitation, is the low pressure zone of below vapour pressure.

A time-dependant pitching oscillation of the hydrofoil was implemented as a numerical test cycle for different hydrofoil profiles. CFX was used to solve the Unsteady Navier-Stokes equations (URANS) with a two-equation turbulence model (Shear Stress Transport SST). A single motion cycle was simulated, incorporating a slow angle of attack variation around nominal conditions followed by a single upstroke pitch cycle, increasing the angle of attack until dynamic stall appears, followed by a subsequent downstroke pitch cycle. The fast downstroke following the high maximum angle leads to flow separation at the leading edge of the baseline profile.

Evolutionary algorithms, as implemented in the EASY tool from National Technical University of Athens (NTUA) [7], were used to identify Pareto-optimal profiles amongst a class derived by Bezier-curves-based deformations of the baseline profile. This solution is preferred mainly for its robustness, easy interconnection with existing virtual test rigs and parallelization possibilities. Only the upstream part of the profile is parameterized, whereas the downstream part is left unmodified. Such a procedure allows the application of optimization results on existing profiles, and easily ensures the structural integrity of the foil. The meshes for performance evaluation were obtained by purely algebraic deformation routines incorporating also mesh deformation for the pitching cycle (ALE moving mesh approach). The first generation is populated covering the parameter space stochastically. Then for the following generations, parameters of the best individuals of a generation (the "elite") are combined while maintaining a certain amount of randomness ("mutation"). Best individuals can remain in the elite population over several generations. Main parameters are:

- Offspring population size (60)

- Parent population size (25)

- Maximum elite population size (20)

The high-performance computer (HPC) cluster, Pleiades, was used to perform parallel computation of these numerous evaluations. The Pareto-front showed that it is possible to maximize lift-to-drag ratio while maintaining sufficient margins for both flow separation and cavitation. When striving for profiles with even higher lift-to-drag ratios (typically having sharp trailing edges), the margins decrease. The conclusion is that the best attainable compromise appears to be characterized by a strongly asymmetric leading edge, which gives rise to a regular curvature of flow pathlines, even under high incidence angles. 
The 2D Pareto-optimal profiles thus identified could serve as a basis for design improvements of the $3 \mathrm{D}$ lifting hydrofoils.

\section{4 (c) Validations of Unsteady Flow Simulations}

As an a posteriori validation of the obtained results, an initial profile and a chosen Pareto-optimal solution were investigated experimentally using stationary lift and drag measurements. Experiments qualitatively confirm the significant differences between the flow around the two profiles, and that the increase in drag coefficient is postponed to higher angles of attack for the profile with the leading edge bend downwards. Nevertheless, absolute quantitative comparison of key parameters is not entirely satisfactory, particularly for the drag coefficient (Figure 16), and for the stall angle. Trends predicted with such numerical simulations therefore have to be treated only as valuable indicators of relative performance when comparing profiles in optimization methods. Experimental investigations remain mandatory to validate the trends and to make reliable statements about the development of cavitation, where modeling uncertainty is even more severe.

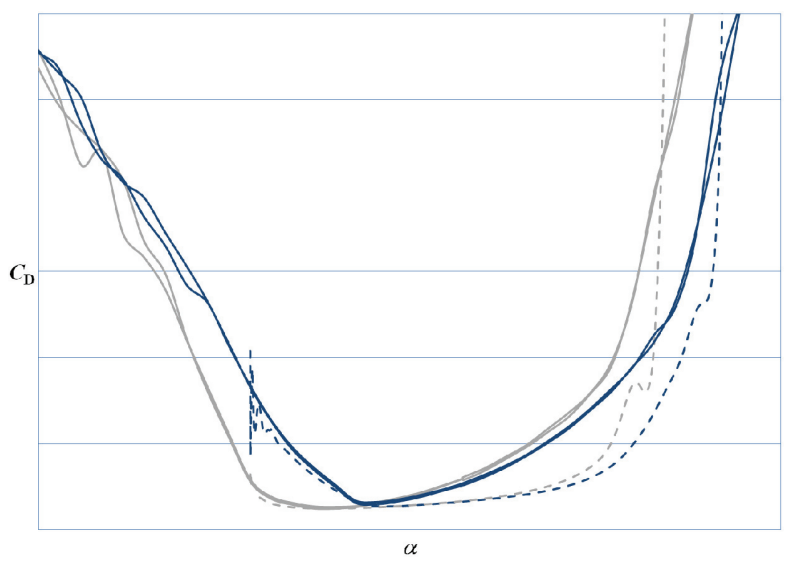

Figure 16: Profile drag coefficient; comparison between initial profile (gray) and Pareto-optimal profile (dark blue) (- - URANS simulation, - experiment)

\subsection{LARGE EDDY SIMULATION}

Since validation studies revealed the lack of quantitative agreement between experiments and URANS simulations, the analysis of the hydrofoil was refined using Large Eddy Simulation (LES). Since the large scales of the flow are computed with this methodology, new phenomena inducing drag should be captured. Instead of using one of the previous specific profiles, this study moved on classical NACA0009 profile, after revealing the same behaviour tendencies and defects within simulation-experiment correlations. LES simulations revealed unsteady detached flows and vortex streets from the leading edge far before stall, and even before small detached trailing edge bubbles predicted by URANS simulations.
Since detached zones and vortices correspond to low pressure zones, a first approach to confirm these numerical results was simple cavitation visualization. A second approach consisted of determining the whole velocity field in the flow near the foil using Particle Image Velocimetry (PIV) [8], thus avoiding the generation of cavitation and the associated risk of flow modifications. The analysis of experimental data revealed large vortex structures in agreement with LES simulations (Figure 17)

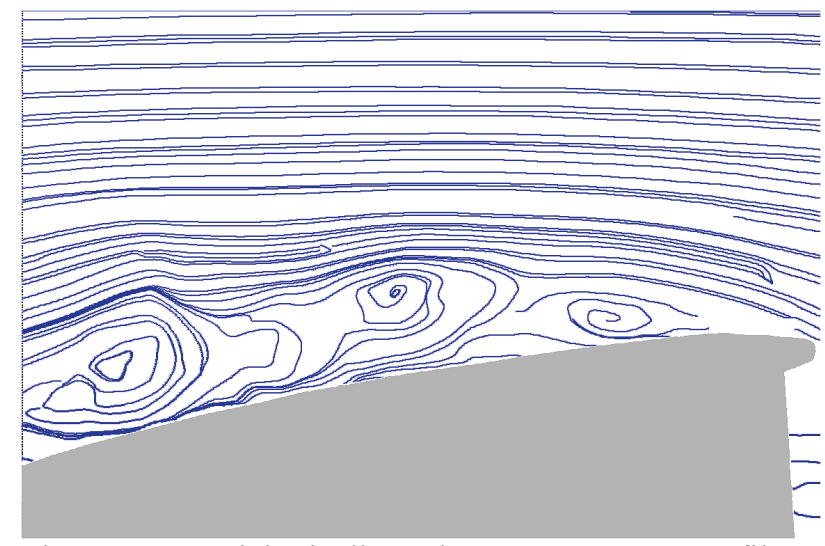

Figure 17: Vorticity isolines above NACA0009 profile

\section{PERSPECTIVES}

Avoiding software licensing constraints enables optimization tasks to use more efficiently the available HPC resources. The accuracy of open-source simulation software solutions, e.g. based on the OpenFOAM libraries [9], will be evaluated in comparison with former numerical studies and experiments on hydrofoils carried out in the hydrodynamic tunnel.

Fluid-structure interaction studies will be extended in two main directions:

- Investigation of instabilities (divergence, flutter) taking into account initially the foil alone, and subsequently the whole platform

- Dynamic behaviour of sandwich structures in waves (slamming)

The computer vision system for the detection of the cross-beam torsion will be finalized. Moreover, other measuring systems such as fiber Bragg grating sensors (FBGS) will be tested, and advanced monitoring tools will be developed.

The computer vision tools could be redirected towards alarms to ensure the safety of the boat during long duration sailing. In particular, rudder/stabilizer immersion detection or cross beam torsion identification could be used to prevent capsizing. 


\section{CONCLUSIONS}

In direct association with the Hydroptère Design Team, several laboratories at EPFL have contributed to a number of optimizations of l'Hydroptère that have enabled the sailing team to achieve the world sailing speed records. Several FE models of the structure have been developed and updated. Specific materials have also been developed, produced and tested in various conditions with the aim to optimize the structural behaviour and provide new design solutions. Onboard measurements of structural deformation and foil immersion used to validate and refine the design loads and operation scenarios have proved to be very important in the fine optimization of the yacht. Various foils were tested at reduced scale in a high-speed water tunnel, and the results used to validate numerical simulations. Globally, using the results of a variety of design-oriented studies by the involved laboratories, the Hydroptère Design Team has been able to address a number of issues in a quick, efficient and interactive manner.

The collaboration between l'Hydroptère and EPFL continues with further challenging objectives for both sailors and researchers. Several studies have already been directed towards the design and optimization of the smaller l'Hydroptère.ch catamaran that will soon be launched on Lake Geneva. Initial reflections regarding the larger l'Hydroptère Maxi are also currently in progress.

\section{ACKNOWLEDGEMENTS}

L'Hydroptère and this scientific collaboration with the EPFL are supported by Thierry Lombard, Lombard Odier Darier Hentsch \& Cie, and Audemars Piguet.

EASY, an optimization tool developed at the Turbomachinery Laboratory at NTUA Athens, was kindly provided by Prof. Giannakoglu and his scientific collaborators.

Finally, we should acknowledge all the students who have contributed to many stages of this collaboration, among: M. Tinguely, M. Reclari, D. Burgstaller, A. Ratouis, P. Gaillard, O. Pacot, C. Dufour, C. Legris, R. Gubler, M. Henry, K. Lilla, J. Michalik, L. Monnard, J. Ostlund, A. Varol, F. Dujonc, G.A. Benvenuti, L. Genolet, M. Ferrario, C. Brière, B. Golaz...

\section{REFERENCES}

1. FLUENT and CFX User's Manuals, ANSYS, Inc., USA http://www.ansys.com/

2. Abaqus User's Manual, Dassault Systèmes SIMULIA, USA http://www.simulia.com/
3. KULLBACK, S., and LEIBLER, R. A., 'On Information and Sufficiency', The Annals of Mathematical Statistics, 22(1):79-86, 1951

4. BRENNEN, C. E., 'Cavitation and Bubble Dynamics', Oxford University Press, USA, 1995 http://resolver.caltech.edu/CaltechBOOK:1995.001

5. AVELLAN, F., HENRY, P., and RYHMING, I.L., 'A New High Speed Cavitation Tunnel', ASME Winter Annual Meeting, Boston, MA, 57:49-60, 1987

6. HOERNER, S. F., 'Fluid-dynamic drag', 1965

7. GIANNAKOGLOU, K. C., 'Design of Optimal Aerodynamic Shapes Using Stochastic Optimization Methods and Computational Intelligence', Progress in Aerospace Sciences, 38(1):43-76, 2002 http://velos0.1tt.mech.ntua.gr/EASY/

8. FlowMap PIV Installation and User's Guide, Dantec Dynamics A/S, Denmark, 2000 http://www.dantecdynamics.com/

9. WELlER, H.G., TABOR, G., JASAK, H., and FUREBY, C., 'A Tensorial Approach to Computational Continuum Mechanics Using Object Oriented Techniques, Computers in Physics, 12(6):620-631, 1998

http://www.openfoam.com/

\section{AUTHORS BIOGRAPHY}

Martin Calmon and Mohamed Farhat are respectively Assistant and Senior Scientist at the Hydraulic Machines Laboratory (LMH) of the Ecole Polytechnique Fédérale de Lausanne (EPFL). Mohamed Farhat is heading the research group on cavitation and interface phenomena. http://1mh.epfl.ch/

Pascal Fua, Konstantin Startchev and Guillaume Bonnier are respectively Director, Engineer and Assistant at the Computer Vision Laboratory (CVLab) of the EPFL.

http://cvlab.epfl.ch/

Jan-Anders Månson, Véronique Michaud, Antoine Sigg and Marc Oggier are respectively Director, Professor and Assistants at the Laboratory of Polymer and Composite Technology (LTC) of the EPFL. http://1tc.epfl.ch/

Michel Deville, Olivier Braun and Mark Sawley are respectively Professor Emeritus (former Director) and Scientists at the Computational Engineering Laboratory (LIN) of the EPFL http://lin.epfl.ch/ 
Luc Blecha and Joël Cugnoni are respectively Postdoctoral Researcher and Research Associate at the Laboratory of Applied Mechanics and Reliability Analysis (LMAF) of the EPFL. Luc Blecha is now Chief Technical Officer at Almatech.

http://lmaf.epfl.ch/

Jean-Mathieu Bourgeon is R\&D Manager, Stéphane Dyen and Davy Moyon are Fluid Dynamics Engineers, Daniel Schmäh is Composite Materials Engineer, Robin Amacher is Naval Architect, and Damien Colegrave is Test Engineer at Hydroptère SA.

http://www.hydroptere.com/ 